\title{
Reversal of Trimethyltin-Induced Learning and Memory Deficits by 3,5-Dicaffeoylquinic Acid
}

\author{
Jin Yong Kang, ${ }^{1}$ Seon Kyeong Park, ${ }^{1}$ Tian Jiao Guo, ${ }^{1}$ Jeong Su Ha, ${ }^{1}$ Du Sang Lee, \\ Jong Min Kim, ${ }^{1}$ Uk Lee, ${ }^{2}$ Dae Ok Kim, ${ }^{3}$ and Ho Jin Heo ${ }^{1}$ \\ ${ }^{1}$ Division of Applied Life Science (BK21 Plus), Institute of Agriculture and Life Science, Gyeongsang National University \\ Jinju 52828, Republic of Korea \\ ${ }^{2}$ Division of Special Purpose Tree, National Institute of Forest Science, Suwon 16631, Republic of Korea \\ ${ }^{3}$ Department of Food Science and Biotechnology, Kyung Hee University, Yongin 17104, Republic of Korea
}

Correspondence should be addressed to Ho Jin Heo; hjher@gnu.ac.kr

Received 6 July 2016; Accepted 21 November 2016

Academic Editor: Byoung-Joon Song

Copyright (c) 2016 Jin Yong Kang et al. This is an open access article distributed under the Creative Commons Attribution License, which permits unrestricted use, distribution, and reproduction in any medium, provided the original work is properly cited.

\begin{abstract}
The antiamnesic effect of 3,5-dicaffeoylquinic acid (3,5-diCQA) as the main phenolic compound in Artemisia argyi $\mathrm{H}$. extract on cognitive dysfunction induced by trimethyltin (TMT) $(7.1 \mu \mathrm{g} / \mathrm{kg}$ of body weight; intraperitoneal injection) was investigated in order to assess its ameliorating function in mice. In several behavioral tests, namely, the Y-maze, passive avoidance, and Morris water maze (MWM) test, 3,5-diCQA significantly ameliorated learning and memory deficits. After the behavioral tests, brain tissues from the mice were analyzed to characterize the basis of the neuroprotective effect. Acetylcholine (ACh) levels increased, whereas the activity of acetylcholinesterase (AChE) decreased upon administration of 3,5-diCQA. In addition, 3,5-diCQA effectively protected against an increase in malondialdehyde (MDA) content, an increase in the oxidized glutathione (GSH) ratio, and a decline of total superoxide dismutase (SOD) level. 3,5-diCQA may prevent neuronal apoptosis through the protection of mitochondrial activities and the repression of apoptotic signaling molecules such as p-Akt, BAX, and p-tau (Ser 404).
\end{abstract}

\section{Introduction}

Alzheimer's disease $(\mathrm{AD})$ is a type of dementia associated with multiple etiologies and pathogenetic mechanisms such as genetic factors, diminished cerebral energy metabolism, excitotoxic events, and free-radical-induced oxidative stress. Among them, oxidative stress is the most important cause in $\mathrm{AD}$ [1]. Oxidative stress occurs due to an excess of oxidants, which is caused by an imbalance between oxidants such as reactive oxygen species (ROS) and antioxidants (e.g., catalase, SOD, and GSH). Oxidants form a normal product of aerobic metabolism, but under pathophysiological conditions oxidants are produced rapidly [2]. Oxidative stress creates numerous problems, such as a decline of antioxidants and an increase of free iron in the brain, and leads to mitochondrial cytopathies [3]. Accordingly, attention to antioxidants has increased, and phenolic compounds have been studied as a source of natural antioxidants. Over 10,000 phenolic compounds are known to be present in the various plants. Polyphenols are essential to plant physiology and belong to the class of natural antioxidants [4]. According to the research by Stocker [5], dietary polyphenols have biological effects such as free-radical scavenging, metal modulation of enzymatic activity, and alteration of signal transduction.

Dicaffeoylquinic acids (diCQAs) as isochlorogenic acid, being natural phenolic compounds, are widely distributed in plants such as Gynura [6] and coffee bean [7]. They are esters formed from quinic acid and two units of caffeic acid. It has been reported that 3,5-dicaffeoylquinic acid (3,5diCQA), one of the diCQA compounds, possesses strong antimutagenic [8], antioxidant [9], and anti-inflammatory activity [10]. Further, Kim et al. have reported that 3,5diCQA possesses a cytoprotective effect against hydrogen peroxide-induced oxidative stress [11]. However, there is no research into in vivo antiamnesic effect of 3,5-diCQA, and, in 
particular, the effect of 3,5-diCQA on the apoptotic signaling pathway has not yet been reported.

Trimethyltin (TMT) is a potent neurotoxicant acting in the hippocampus, and intraperitoneal injection of TMT increased ROS production rate in mice in sensitive areas such as the hippocampus and the frontal cortex, and the increased level of ROS induced oxidative damage, which contributes to activate the apoptotic signaling pathway [12]. We therefore investigated the ameliorating effect of 3,5-diCQA on TMT-induced learning and memory impairment in ICR mice through an examination of antioxidant biochemicals, mitochondrial activity, and apoptotic signaling molecules in brain tissues.

\section{Materials and Methods}

2.1. Chemicals and Reagents. A 3,5-diCQA (PubChem CID: 6474310) was purchased from Chengdu Biopurify Phytochemicals Ltd. (Chengdu, China). TMT, dimethyl sulfoxide (DMSO), metaphosphoric acid, thiobarbituric acid (TBA), sodium azide, Triton X-100, Tween 20, 5, $5^{\prime}$-dithiobis(2-nitrobenzoic acid) (DTNB), and all other chemicals were purchased from Sigma-Aldrich Chemical Co. (St. Louis, MO, USA). Primary antibodies for cytochrome c (sc-13560), ptau (Ser 404) (sc-12952), and $\beta$-actin (sc-69879) were purchased from Santa Cruz Biotechnology (CA, USA). Primary antibodies for protein kinase B (Akt) (\# 9272), phospho-Akt (Ser 473) (\# 9271), and BAX (\#2772) and secondary antibodies for anti-rabbit (7074S) and anti-mouse (7076S) were obtained from Cell Signaling Technology (Danvers, MA, USA).

\subsection{Sample Preparation and Ultra-Performance Liquid Chro-} matography-Quadrupole-Time-of-Flight (UPLC-Q-TOF) MS Analysis. Artemisia argyi $\mathrm{H} .(20 \mathrm{~g})$ was extracted with $60 \%$ ethanol $(1 \mathrm{~L})$ at $40^{\circ} \mathrm{C}$ for $2 \mathrm{~h}$ using reflux extraction. The ethanolic extract was partitioned with $n$-hexane, chloroform, and ethyl acetate, consecutively, and then each fraction was concentrated and lyophilized.

Phenolic compounds extracted from Artemisia argyi $\mathrm{H}$. were analyzed by using UPLC-Q-TOF/MS (Waters, Milford, MA, USA). The samples were dissolved in methanol after that filtered using $0.2 \mu \mathrm{m}$ filter and then injected into an Acquity UPLC BEH C ${ }_{18}$ column $(100 \times 2.1 \mathrm{~mm}, 1.7 \mu \mathrm{m}$; Waters $)$ with a flow rate of $0.4 \mathrm{~mL} / \mathrm{min}$. The mobile phases consisted of solvent A (distilled water containing $0.1 \%$ formic acid) and solvent B (acetonitrile (ACN) containing $0.1 \%$ formic acid) during analysis. The analysis conditions were as follows: a gradient elution of $100 \% \mathrm{~A} / 0 \% \mathrm{~B}$ at $0.5 \mathrm{~min}, 0 \% \mathrm{~A} / 100 \% \mathrm{~B}$ at $5 \mathrm{~min}, 0 \% \mathrm{~A} / 100 \% \mathrm{~B}$ at $6.5 \mathrm{~min}, 100 \% \mathrm{~A} / 0 \% \mathrm{~B}$ at $6.8 \mathrm{~min}$, and $100 \% \mathrm{~A} / 0 \% \mathrm{~B}$ at $9 \mathrm{~min}$. Phenolic compounds were analyzed by a Q-TOF MS (Waters) in electrospray ionization- (ESI-) negative mode. The conditions used for the ESI source were as follows: ramp collision energy, $20-45 \mathrm{~V}$; oven temperature, $40^{\circ} \mathrm{C}$; capillary voltage, $3 \mathrm{kV}$; desolvation temperature, $350^{\circ} \mathrm{C}$; pressure of nebulizer, $40 \mathrm{psi}$; fragmentor, $175 \mathrm{~V}$; cone voltage, $40 \mathrm{~V}$; mass range, $50-1200 \mathrm{~m} / z$. All MS data obtained by MassLynx software (Waters), including retention time (RT), $\mathrm{m} / z$, and ion intensity, were extracted with MarkerLynx software (Waters).
2.3. Animals and In Vivo Experimental Design. Institute of Cancer Research (ICR) male mice (age, 4 weeks) were obtained from Samtako (Osan, Korea) and were housed two per cage at a temperature of $25^{\circ} \mathrm{C}$ with a $12 \mathrm{~h}$ light-dark cycle, relative humidity of $55 \%$, and free access to food and water ad libitum. All animal procedures were carried out as required by the "Institutional Animal Care and Use Committee" of Gyeongsang National University (certificate: GNU131105-M0067). Mice were (orally) fed 3,5-diCQA as main phenolic compounds of Artemisia argyi $\mathrm{H}$. at concentrations of $5 \mathrm{mg} / \mathrm{kg}$ of body weight (3,5-diCQA 5 group) and $10 \mathrm{mg} / \mathrm{kg}$ of body weight (3,5-diCQA 10 group) once a day for 3 weeks. After administration of 3,5-diCQA for 3 weeks, TMT $(7.1 \mu \mathrm{g} / \mathrm{kg}$ of body weight) was dissolved in $0.85 \%$ sodium chloride solution and injected into mice intraperitoneally $(100 \mu \mathrm{L})$, except for mice in the vehicle control group. Mice of TMT group were injected with only TMT.

\subsection{Behavioral Tests for Learning and Memory Function.} After 3 days of TMT injection, a Y-maze test was conducted. The maze was made from black Y-shaped plastic, and the arms were at an angle of $120^{\circ}$ from each other. Each mouse could freely move in the maze for $8 \mathrm{~min}$, and the sequence of arm entries was recorded with a Smart 3.0 video tracking system (Panlab, Barcelona, Spain). The percentage spatial cognition ability was calculated as follows: actual alternation/(total number of arm entries -2$) \times 100(\%)$ [13].

After the Y-maze test, the passive avoidance test was conducted on 2 days. The apparatus consisted of a two-compartment acrylic box, one compartment illuminated and the other dark, with a gate between the two compartments. On the first day of the experiment, each mouse was placed in the lit compartment. As soon as it entered the dark compartment, it received an electrical shock $(0.5 \mathrm{~mA}, 3 \mathrm{~s})$. After $24 \mathrm{~h}$, the latency times for entering the dark compartment were measured for maximum $300 \mathrm{~s}$ [14].

The Morris water maze (MWM) test was conducted in accordance with Morris but with some modifications [15]. The apparatus consisted of a large circular pool $(90 \mathrm{~cm}$ in diameter) containing opaque water dyed with squid ink (Cebesa, Valencia, Spain) at $22 \pm 2^{\circ} \mathrm{C}$. The circular pool was divided into quadrants ( $\mathrm{N}, \mathrm{S}, \mathrm{E}$, and $\mathrm{W}$ zones) with visual cues on the walls for navigation, and a platform was located in the $\mathrm{W}$ zone. Training was conducted by means of four trials daily for 4 consecutive days. For each trial, mice were placed in the water facing the wall of the pool but at different start positions between trials. Mice were trained to find the platform for a maximum of $60 \mathrm{~s}$. After training was complete, the platform was removed and behavior of the mice was recorded for $60 \mathrm{~s}$ using a Smart 3.0 video tracking system (Panlab, Barcelona, Spain).

2.5. Tissue Preparation and Biochemical Studies. After the behavioral tests, the mice were sacrificed by $\mathrm{CO}_{2}$ inhalation, and brain tissues were kept at $-80^{\circ} \mathrm{C}$ until they were used. Whole brain tissue was minced into small pieces and then homogenized with 10 volumes of cold phosphate buffered saline for the measurement of MDA, AChE, ACh, and total SOD levels. To measure oxidized GSH and total GSH, minced 
brain tissues were homogenized with 20 volumes of $5 \%$ metaphosphoric acid.

The quantity of ACh neurotransmitter in the brain was estimated by the method of Vincent et al. Homogenates were centrifuged $\left(14,000 \times \mathrm{g}\right.$ for $30 \mathrm{~min}$ at $\left.4^{\circ} \mathrm{C}\right)$ to obtain the supernatant [16]. Alkaline hydroxylamine reagent [3.5 N sodium hydroxide and $2 \mathrm{M}$ hydroxylamine in $\mathrm{HCl}$ ] was added to the supernatant. After $1 \mathrm{~min}$ at room temperature, $0.5 \mathrm{~N} \mathrm{HCl}(\mathrm{pH} 1.2)$ and $0.37 \mathrm{M} \mathrm{FeCl}_{3}$ in $0.1 \mathrm{~N} \mathrm{HCl}$ were added, and absorbance was measured at $540 \mathrm{~nm}$. AChE was measured via ACh hydrolyzing activity following Ellman's method [17]. Mixtures of brain homogenate and $50 \mathrm{mM}$ sodium phosphate buffer $(\mathrm{pH} 8.0)$ were incubated at $37^{\circ} \mathrm{C}$ for $15 \mathrm{~min}$ and then added to Ellman's reaction mixture $[0.5 \mathrm{mM}$ acetylthiocholine and $1 \mathrm{mM}$ DTNB] in a $50 \mathrm{mM}$ sodium phosphate buffer $(\mathrm{pH} 8.0)$. Absorbance was measured at $405 \mathrm{~nm}$ after incubation at $37^{\circ} \mathrm{C}(20 \mathrm{~min})$.

To measure MDA as a marker of lipid peroxidation, homogenates were centrifuged $\left(6000 \times \mathrm{g}\right.$ for $10 \mathrm{~min}$ at $\left.4^{\circ} \mathrm{C}\right)$, and the supernatant was mixed with $0.67 \%$ TBA solution with $1 \%$ phosphoric acid and then incubated in a water bath $\left(95^{\circ} \mathrm{C}\right)$ for $1 \mathrm{~h}$. After cooling, absorbance was measured at $532 \mathrm{~nm}$ [18].

Homogenates for measuring total SOD were centrifuged at $400 \times \mathrm{g}$ for $10 \mathrm{~min}$ at $4^{\circ} \mathrm{C}$, and then 10 volumes of $1 \mathrm{x}$ Cell Extraction Buffer $[10 \%$ SOD buffer, $0.4 \%(\mathrm{v} / \mathrm{v})$ Triton X100 , and $200 \mathrm{Mm}$ phenylmethanesulfonyl fluoride in distilled water] were added to the pellet. Mixtures were incubated on ice for $30 \mathrm{~min}$ and then centrifuged at $10,000 \times \mathrm{g}$ for $10 \mathrm{~min}$ at $4^{\circ} \mathrm{C}$ to obtain the supernatant. The SOD content of supernatant was measured with the SOD kit of Sigma-Aldrich Chemical Co.

Homogenates for measuring the ratio of oxidized GSH/ total GSH were centrifuged at $14,000 \times \mathrm{g}$ for $15 \mathrm{~min}$ at $4^{\circ} \mathrm{C}$. Oxidized GSH/total GSH ratios in the supernatant were measured with the glutathione (oxidized GSH/total GSH) detection kit of Enzo Life Science Inc. (Enzo Diagnostics, NY, USA).

2.6. Isolation of Mitochondria from Brain Tissues and Measurement of Mitochondrial Activities. Mitochondria were isolated in accordance with the procedure of Dragicevic [19]. Whole brains were homogenized with 5 volumes of isolation buffer [mannitol $(215 \mathrm{mM})$, sucrose $(75 \mathrm{mM}), 0.1 \%$ bovine serum albumin (BSA) (Bioworld, Dublin, OH, USA), EGTA (1 mM), and $20 \mathrm{mM}$ HEPES $\left(\mathrm{Na}^{+}\right)(\mathrm{pH}$ 7.2)] and then centrifuged at $1,300 \times \mathrm{g}$ for $5 \mathrm{~min}$. After the supernatant was obtained, it was centrifuged once more at $13,000 \times \mathrm{g}$ for $10 \mathrm{~min}$. After this, the supernatant was removed, and isolation buffer with $0.1 \%$ digitonin (in DMSO) was added to the pellet. After $5 \mathrm{~min}$, isolation buffer was added, followed by centrifugation at $13,000 \times \mathrm{g}$ for $15 \mathrm{~min}$. After that, the pellets were resuspended in isolation buffer without EGTA and centrifuged at $10,000 \times \mathrm{g}$ for $10 \mathrm{~min}$. Finally, isolation buffer without EGTA was added to the pellet, and this was used in the experiment.

Mitochondrial ROS production was measured with $2^{\prime}, 7^{\prime}$ dichlorodihydrofluorescein diacetate (DCF-DA). $25 \mu \mathrm{M}$ DCF-DA was added to the isolated mitochondria for $20 \mathrm{~min}$ and then quantified with a fluorescent (excitation filter $485 / 20 \mathrm{~nm}$, emission filter 528/20 nm) microplate reader (Infinite 200, Tecan Co., San Jose, CA, USA) [20].

To measure the membrane potential of isolated mitochondria, $20 \mu \mathrm{L}(1.2 \mathrm{mg} / \mathrm{mL}$ final concentration) of the mitochondria was mixed with assay buffer [isolation buffer without EGTA with pyruvate $(5 \mathrm{mM})$ and malate $(5 \mathrm{mM})]$, and then a solution of $1 \mu \mathrm{M}$ JC-1 $\left(5,5^{\prime}, 6,6^{\prime}\right.$-tetrachloro-1, $1^{\prime}, 3^{\prime}, 3^{\prime}$ tetraethylbenzimi-dazolylcarbocyanine iodide in DMSO) was added. The mixture was gently stirred at room temperature for $20 \mathrm{~min}$ in the dark and then measured with a fluorescent (excitation $530 / 25 \mathrm{~nm}$, emission $590 / 35 \mathrm{~nm}$ ) microplate reader (Infinite 200, Tecan Co.).

Samples of isolated mitochondria were assayed for ATP content by means of the ATP bioluminescence assay kit (Sigma-Aldrich Chemical Co.). ATP concentration was calculated with the help of a standard curve.

2.7. Western Blot Analysis of the Apoptotic Signaling Pathway. Brains were homogenized with ProtinEx ${ }^{\mathrm{TM}}$ Animal cell/tissue (GeneAll Biotechnology, Seoul, Korea) with 1\% protease inhibitor cocktails (Thermo Fisher Scientific, Rockford, IL, USA). The protein samples were separated by sodium dodecyl sulfate polyacrylamide gel electrophoresis (SDS-PAGE) and then transferred to a polyvinylidene difluoride (PVDF) membrane (Millipore, Billerica, MA, USA). The membranes were blocked with $5 \%$ nonfat dry milk in TBST buffer [TrisBuffered Saline (TBS) with $0.1 \%$ of Tween-20]. After $1 \mathrm{~h}$, primary antibodies were diluted $(1: 1000)$ in a dilute solution ( $0.1 \%$ sodium azide and $0.5 \%$ BSA in TBST). Diluted primary antibodies were incubated with the membrane under gentle agitation overnight, and then the membrane was washed 3 times (10 min each time) in TBST. After washing, the secondary antibody solutions were allowed to react with the membrane for $1 \mathrm{~h}$, and then the washing process was carried out once again. Finally, the membrane was exposed to an enhanced chemiluminescence reagent, and the luminescence was detected by ChemiDoc (Korea Biomics, Seoul, Korea). The density of the band was assayed with ImageJ Software (National Institutes of Health, Bethesda, Maryland, USA). The results were presented as density of target protein/density of $\beta$-actin as a loading control [21, 22].

2.8. Statistical Analysis. All results were expressed as mean \pm SD. Each experiment was analyzed by one-way analysis of variance (ANOVA) followed by Duncan's multiple range test with the help of the SAS program (Ver. 9.1 SAS Institute, Cary, NC, USA).

\section{Results and Discussion}

3.1. Phenolic Compounds Analysis Extracted from Artemisia argyi $\mathrm{H}$. Ethyl acetate fractions from Artemisia argyi $\mathrm{H}$., which has most abundant phenolic compounds (Supplementary Data 1 in Supplementary Material avialable online at http://dx.doi.org/10.1155/2016/6981595), were analyzed with a UPLC/Q-TOF-MS/MS system (Figure 1). Main compounds were analyzed by a Q-TOF MS system in ESI-negative mode 
4

Oxidative Medicine and Cellular Longevity

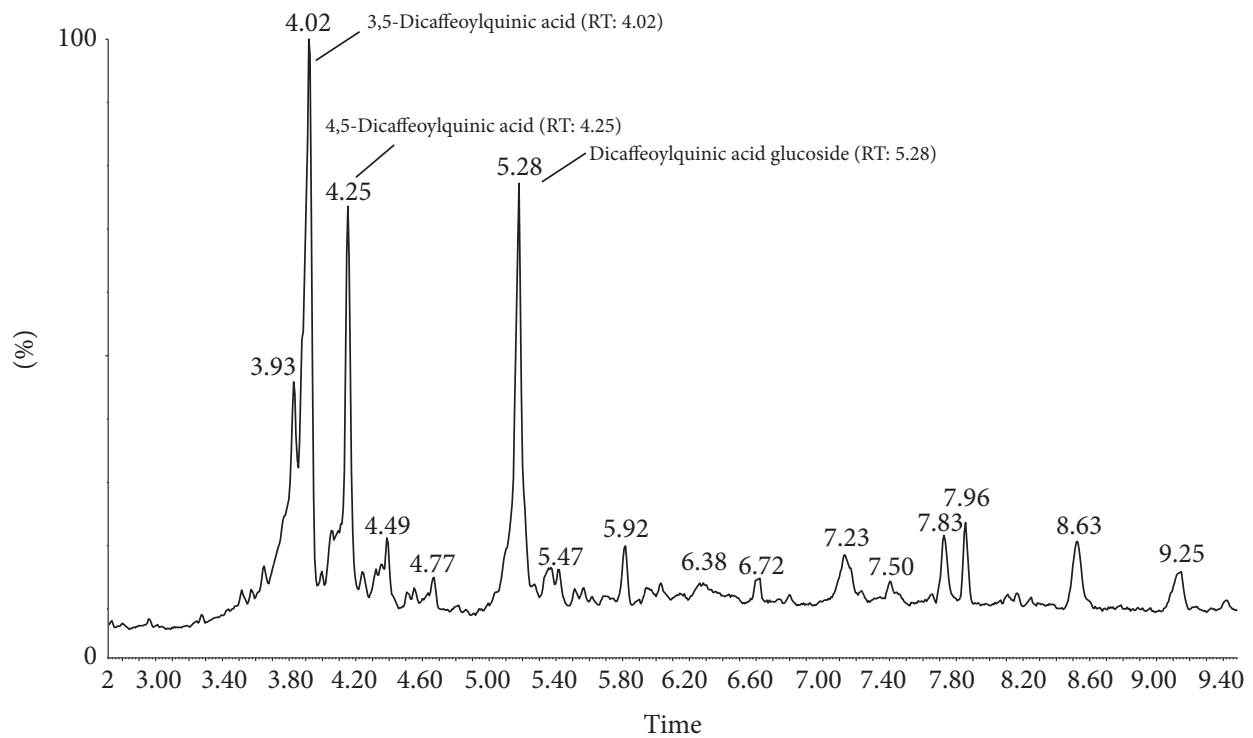

(a)

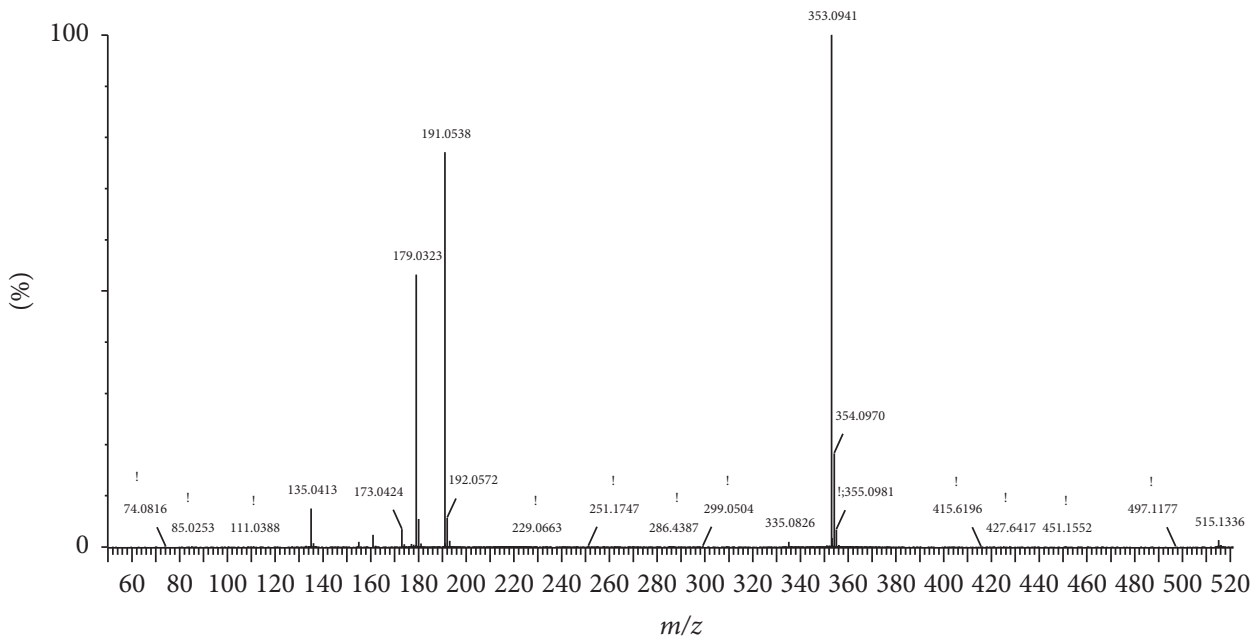

(b)

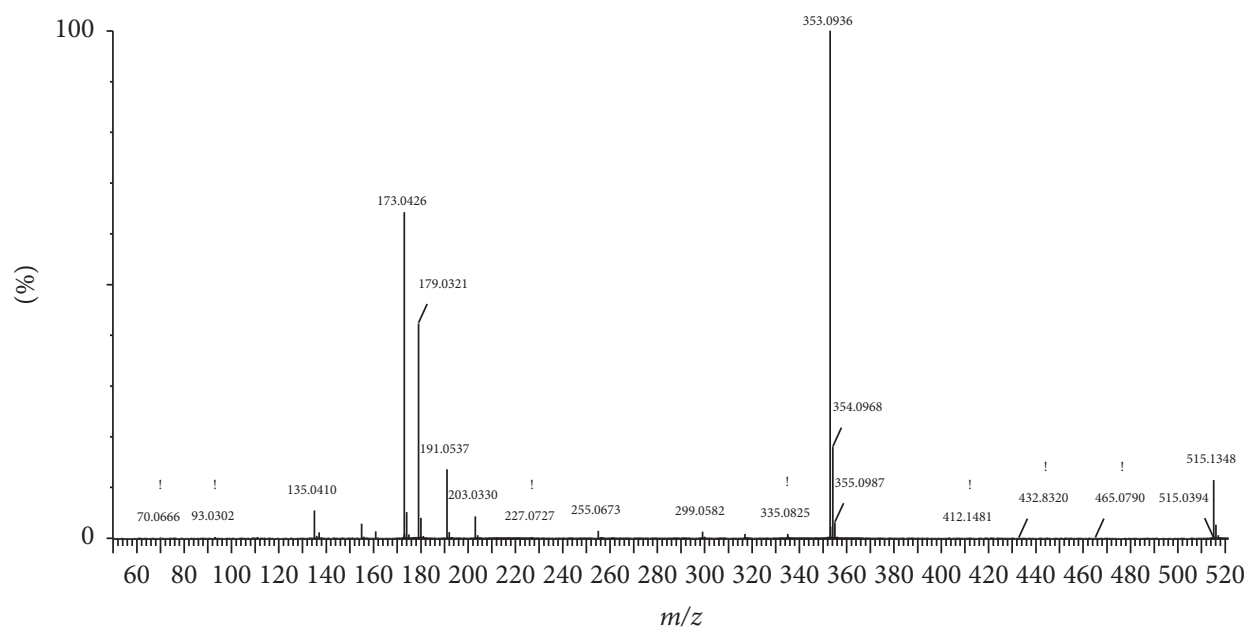

(c)

Figure 1: Continued. 


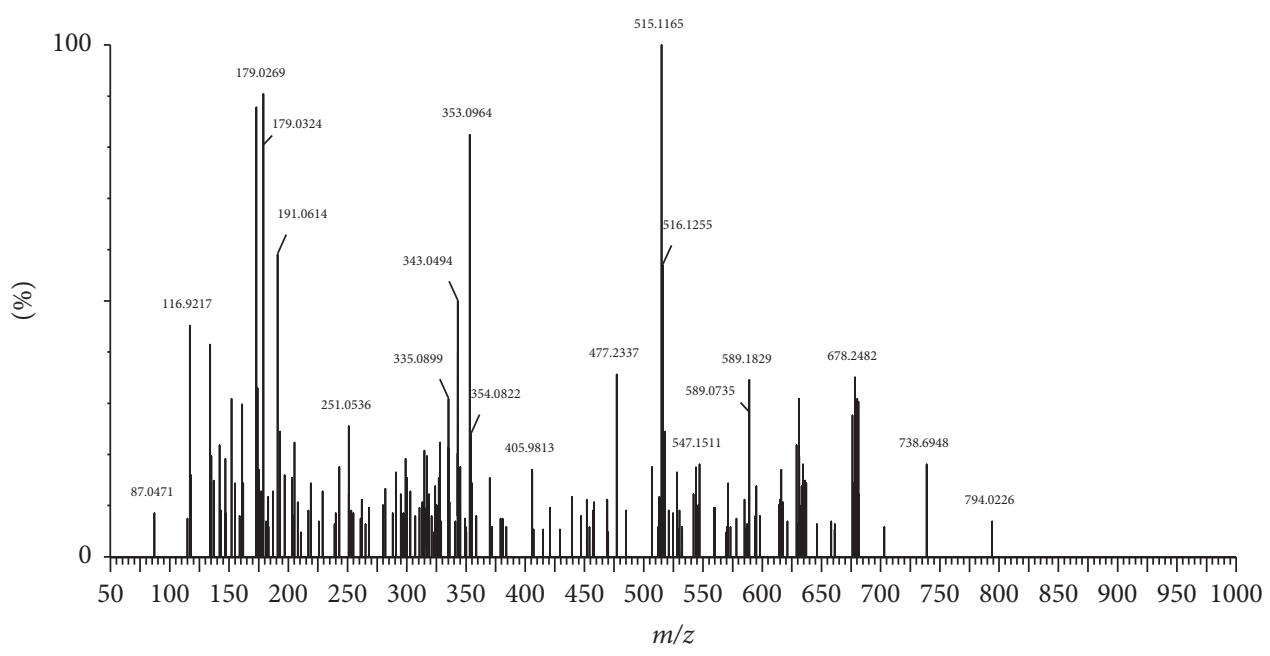

(d)

FIgURE 1: Analysis of ethyl acetate fraction from Artemisia argyi H. using UPLC/Q-TOF-MS/MS chromatography in negative ion mode (a), $\mathrm{MS}^{2}$ patterns of 3,5-diCQA (b), 4,5-diCQA (c), and diCQA-glucoside (d).

(Figures 1(b)-1(d)): compound 1 (RT: $4.02 \mathrm{~min}, 515.13 \mathrm{~m} / z$ ); compound 2 (RT: $4.25 \mathrm{~min}, 515.13 \mathrm{~m} / z$ ); and compound 3 (RT: $5.28 \mathrm{~min}, 677.16 \mathrm{~m} / z$ ). Additionally, $\mathrm{MS}^{2}$ scan chromatograms were fragmented at compound 1 (353.09, 191.05, 179.03 , and $135.04 \mathrm{~m} / z)$, compound $2(353.09,173.04,179.03$, and $191.05 \mathrm{~m} / z)$, and compound $3(515.12,353.09,179.03$, and $191.06 \mathrm{~m} / \mathrm{z}$ ). From comparison to the main fragments in the previous literature [23], these peaks showed the 3,5diCQA (PubChem CID: 6474310), 4,5-diCQA (PubChem CID: 6474309), and diCQA-glucoside. A 3,5-diCQA was identified as the major phenolic compound among 3,5diCQA, 4,5-diCQA, and diCQA-glucoside, and 3,5-diCQA has a higher inhibitory effect on in vitro lipid peroxidation and AChE activity than 4,5-diCQA (Supplementary Data 2).

3.2. Effect of 3,5-diCQA on TMT-Induced Learning and Memory Dysfunction. The Y-maze test was conducted to determine the spatial cognition ability. Figure 2(a) shows the distance travelled by mice in Y-maze test. According to Kim et al., TMT induces hyperactivity disorder [24]. And as a result of hyperactivity, the TMT group exhibited a greater distance travelled than the vehicle control group. On the other hand, in the 3,5-diCQA groups the hyperactivity effect due to TMT was ameliorated. In terms of alternation behavior which is indicative of spatial cognition (Figure 2(b)), the 3,5-diCQA 10 group showed a similar result to the vehicle control group, but the 3,5-diCQA 5 group did not show a significantly different result from the TMT group. Figure 2(c) also shows the behavior pattern of each group during the experimental period, and the improvement of TMTinduced hyperactivity by treatment with 3,5-diCQA was confirmed.

In the passive avoidance test for measuring short-term learning and memory ability, the TMT group had the lowest latency time of all groups as a consequence of TMT-induced learning and memory defects. However, step-through latency increased in both 3,5-diCQA groups. In particular, the 3,5diCQA 10 group showed a similar latency time to the vehicle control group (Figure 2(d)).

Another behavioral test, the MWM test, was conducted to assess long-term learning and memory ability. Figure 3(a) shows escape latency times recorded during sequential training trials. The vehicle control group quickly remembered the correct location of platform in the pool. The escape latency time gradually decreased, and more direct swim paths were taken than in the other groups. In contrast, the TMT group showed the smallest reduction in escape latency time during training. Both 3,5-diCQA groups exhibited improved spatial memory and learning ability, relative to the TMT group. As shown in the probe trials, the 3,5-diCQA groups spent more time in the $\mathrm{W}$ zone, where the platform was located, than the TMT group (Figure 3(b)). In particular, the 3,5-diCQA 10 group spent a similar amount of time in the $\mathrm{W}$ zone as the vehicle control group, and also both 3,5-diCQA groups exhibited improvements over the TMT-induced abnormal travel trajectories (Figure 3(c)). Consequently, a 3,5-diCQA appears to be an effective substance for ameliorating cognitive impairment induced by TMT.

3.3. Effect of 3,5-diCQA on the Cholinergic System in Brain Tissue. ACh acts as a neurotransmitter in the brain. According to the cholinergic hypothesis, reductions in choline acetyltransferase (ChAT) activity and ACh synthesis are closely related with cognitive impairments such as Alzheimer's disease (AD) [25]. Woodruff and Baisden reported that TMT led to a decrease in ChAT and an increase in AChE, an enzyme that catalyzes the breakdown of ACh and some other choline ester neurotransmitters [26]. The TMT group in our experiment similarly showed an increase in AChE activity and a decrease in ACh levels compared with the vehicle control group (Figure 4). Treatment with 3,5-diCQA significantly 


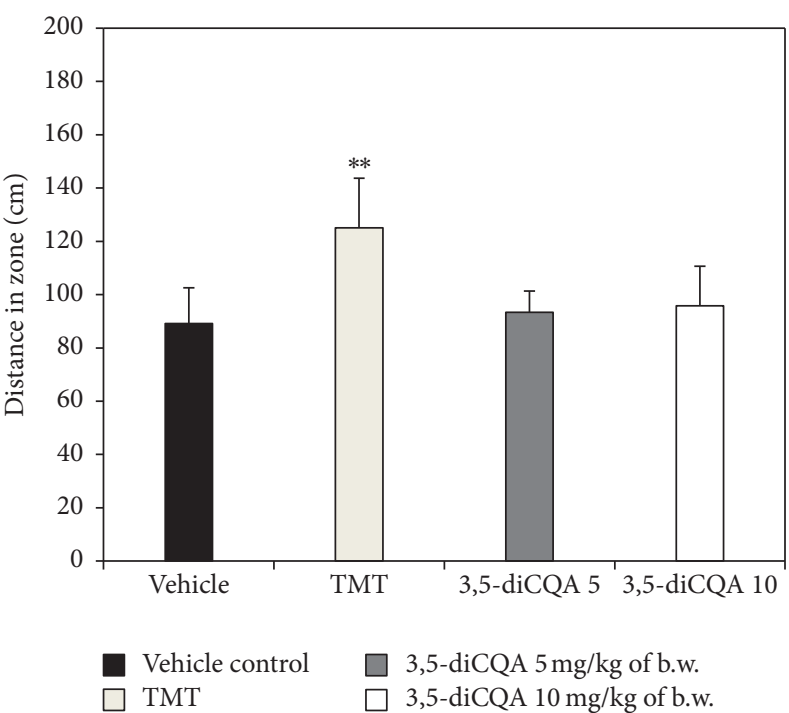

(a)

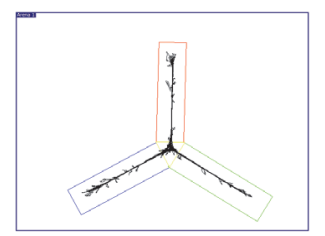

Control

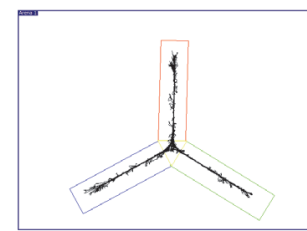

3,5-diCQA 5

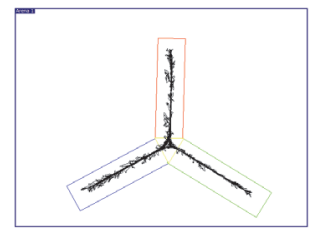

TMT

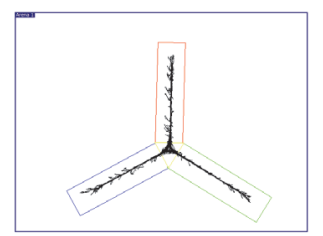

3,5-diCQA 10

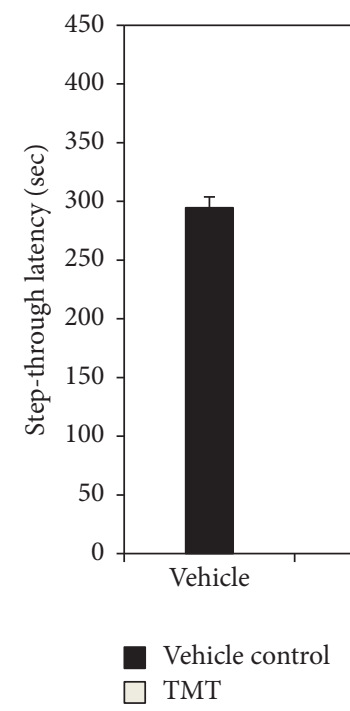

(c)

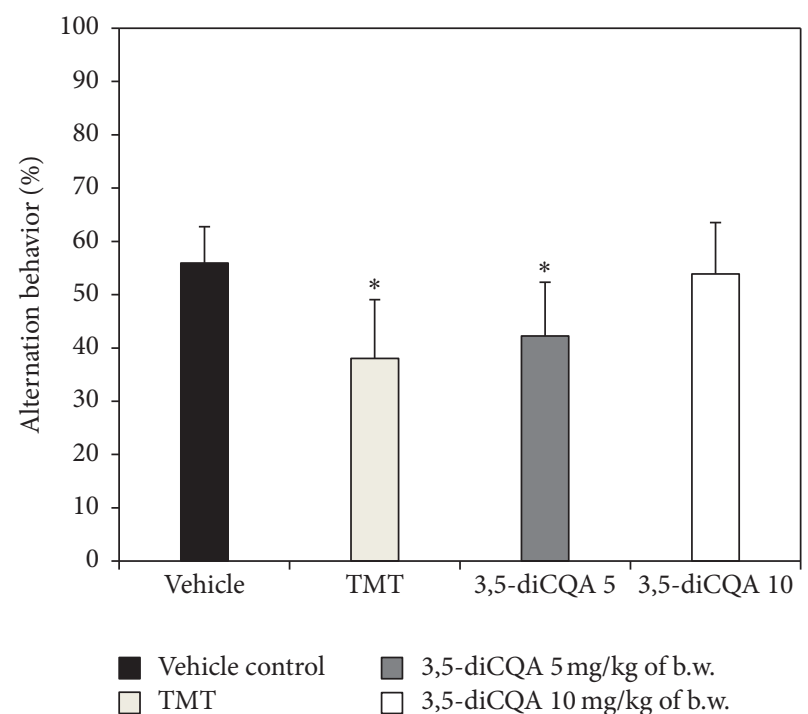

(b) 


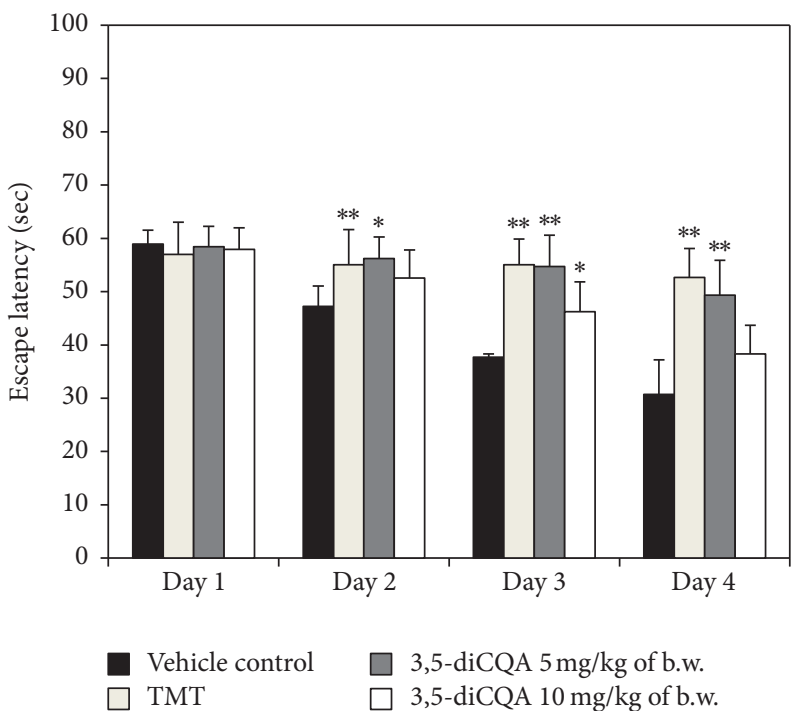

(a)

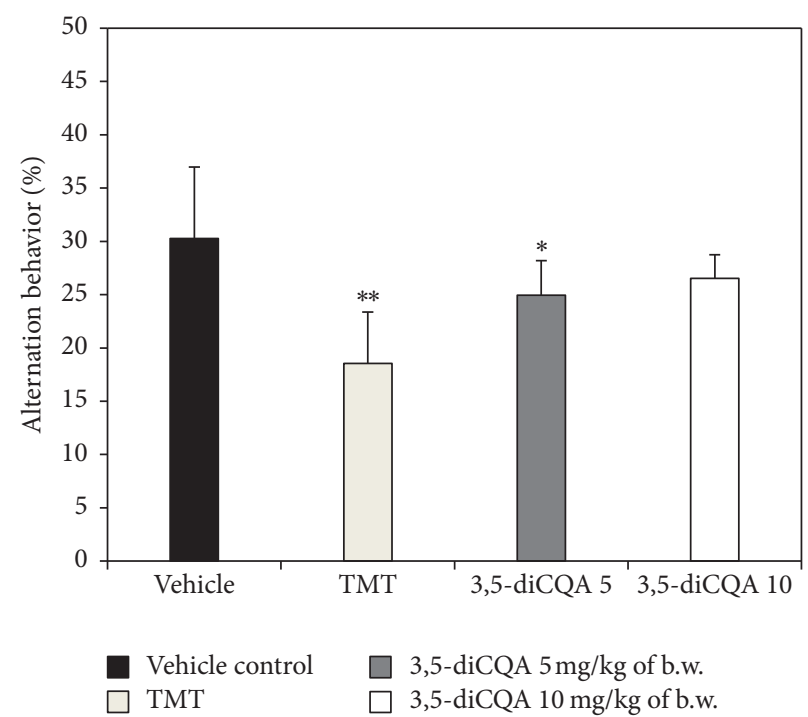

(b)

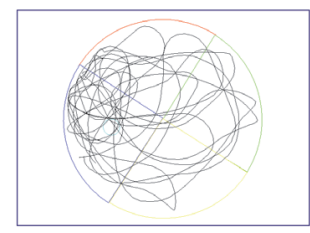

Control

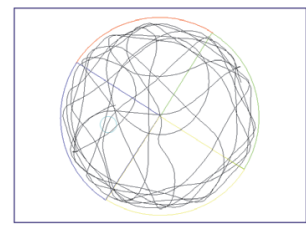

3,5-diCQA 5

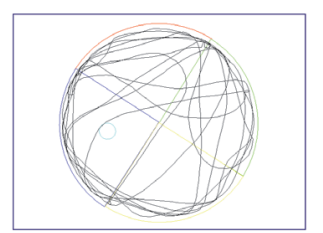

TMT

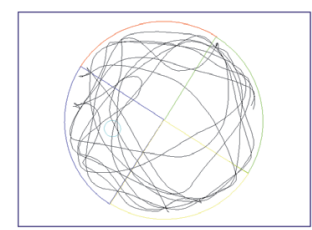

3,5-diCQA 10

(c)

FIGURE 3: Effect of 3,5-diCQA on long-term memory and learning ability on TMT-induced cognitive impairment in MWM test. Escape latency time (a) and time in W zone (b) path of motion in probe test (c). MWM test was conducted during 6 days. Data shown represent means $\pm \mathrm{SD}(n=8) .{ }^{*} P<0.05$ and ${ }^{* *} P<0.01$ compared to the vehicle control group.

used as an indicator of oxidative stress [29]. The oxidized GSH/total GSH ratio in the TMT group was higher than in vehicle control group, and in both 3,5-diCQA groups the ratio was lower than in the TMT group. In other words, the oxidized GSH/total GSH ratio showed a similar pattern as the result of the analysis of MDA levels (Figure 5(b)). The research of Kim et al. has shown that 3,5-diCQA possesses neuroprotective effects against hydrogen peroxide-induced cell death in SH-SY5Y cells as a consequence of inhibiting caspase- 3 activation and restoring GSH levels [11].

According to the previous reports, TMT increases ROS production and oxidative stress due to an excess of oxidants reduces antioxidant levels $[3,12]$. Accordingly, the TMT group in our experiments also exhibited lower SOD levels than the vehicle control group, whereas the SOD contents of both 3,5-diCQA groups were higher than in the TMT group (Figure $5(\mathrm{c})$ ). GSH protects against oxidative stress, and a depletion of GSH is presumably the result of decreased SOD and catalase activities. When the antioxidant mechanism becomes ineffective as a consequence of excessive oxidative stress, lipid peroxidation occurs [30]. In other words, a noticeable depletion of GSH content in brain tissue will lead to an increase lipid peroxidation. Since brain tissue is composed of neuronal cells that contain numerous polyunsaturated fatty acids to carry out various signaling functions, it is vulnerable to lipid peroxidation, and lipid peroxidation in the brain is an indicator of mild cognitive impairment in patients [31]. Oxidative stress induced by TMT may eventually destroy the antioxidant system in brain. Therefore, the collapse of the antioxidant system increased MDA levels and induced cognitive dysfunction. However, these learning difficulties and memory impairments due to TMT-induced cytotoxicity and oxidative stress could be partially recovered by a 3,5-diCQA. 


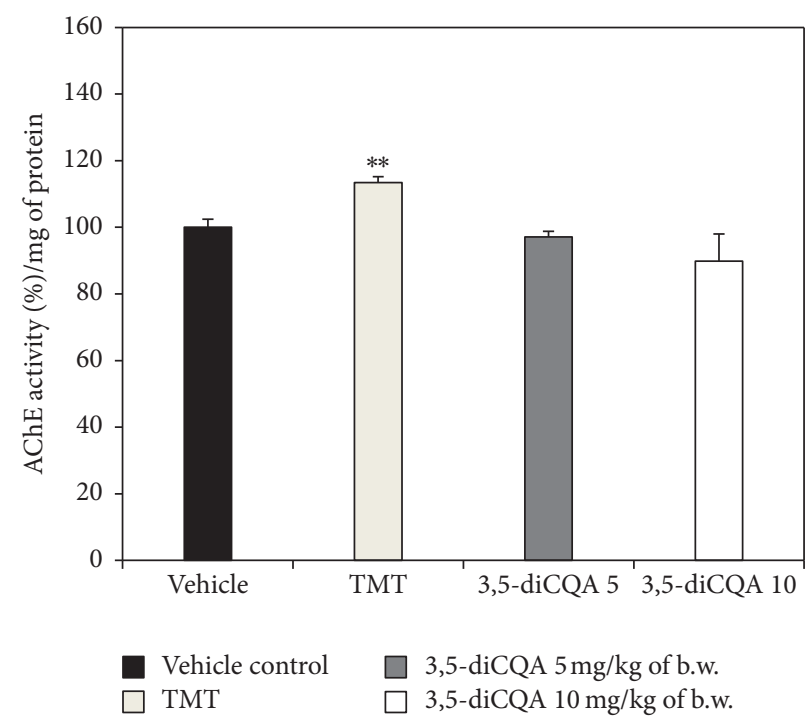

(a)

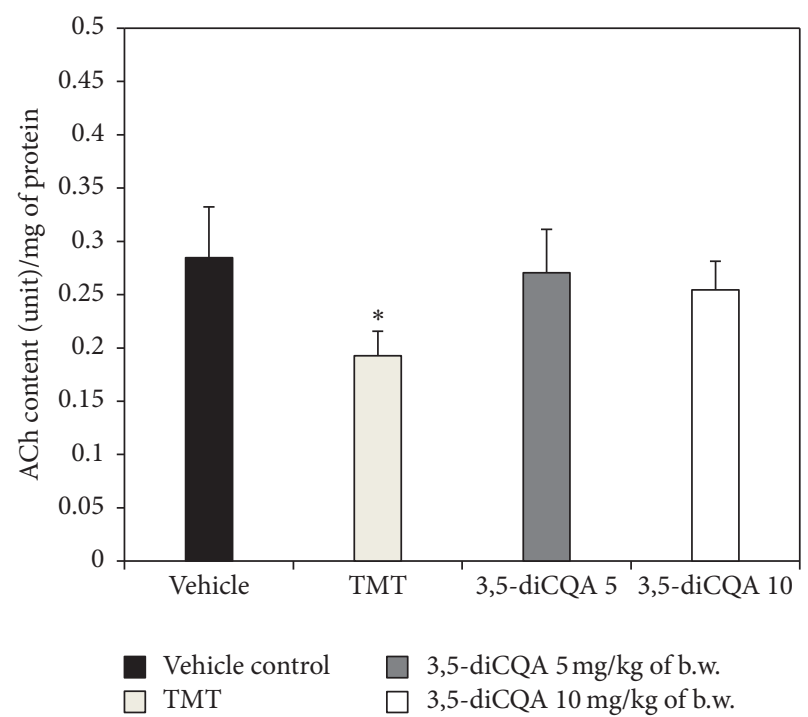

(b)

Figure 4: Effect of 3,5-diCQA on cholinergic markers in TMT injected mice brain. AChE activity (a) and ACh content (b). Data shown represent means $\pm \mathrm{SD}(n=8) .{ }^{*} P<0.05$ and ${ }^{* *} P<0.01$ compared to the vehicle control group.

3.5. Effect of 3,5-diCQA on Mitochondrial Activity in Brain Tissue. Mitochondria are one of the most ROS production organelles in the cell in the pathologic condition. Exposure to excessive oxidative stress results in the opening of one of the requisite mitochondrial channels. This in turn causes the simultaneous collapse of mitochondrial membrane potential and a transient increase in ROS generation [32]. According to the research of Zhang et al., TMT-induced cytotoxicity causes an excessive generation of ROS and a subsequent reduction in mitochondrial membrane potential [33]. Accordingly, increased mitochondrial ROS production and decreased mitochondrial membrane potential were found in the TMT group, compared with vehicle control group. The 3,5-diCQA 5 group did not exhibit significant differences with the TMT group, but the 3,5-diCQA 10 group exhibited reduced mitochondrial ROS production and a higher mitochondrial membrane potential (Figures 6(a) and 6(b)). In other words, TMT-induced oxidative stress led to an increase in mitochondrial ROS production and a decrease mitochondrial membrane potential, while 3,5-diCQA at a concentration of $10 \mathrm{mg} / \mathrm{kg}$ body weight protected mitochondria by reducing TMT-induced oxidative stress.

As the report of Green and Reed reveals, typically an early event in the apoptotic pathway is a rapid reduction of mitochondrial membrane potential which leads to a decrease in ATP content [34]. Accordingly and as expected, ATP levels in the 3,5-diCQA 5 group and TMT group were similar, and ATP levels of 3,5-diCQA 10 group were lower than in the vehicle control group but higher than in the TMT group (Figure 6(c)). Polyphenols could play an important role in mitochondrial biochemistry by modulating antioxidant activity, apoptosis, inflammation, and signal transduction [35], and 3,5-diCQA too is thought to have these functions. Broadly speaking, treatment with 3,5-diCQA at $5 \mathrm{mg} / \mathrm{kg}$ of body weight did not provide sufficient protection against TMT-induced mitochondrial dysfunction. But treatment with 3,5-diCQA at $10 \mathrm{mg} / \mathrm{kg}$ of body weight provided statistically significant protection against TMT-induced mitochondrial dysfunction. In other words, 3,5-diCQA at concentration of $5 \mathrm{mg} / \mathrm{kg}$ body weight could protect the antioxidant system in brain, but only seems to be effective in protecting mitochondria in the brain at a concentration of $10 \mathrm{mg} / \mathrm{kg}$ body weight.

3.6. Effect of 3,5-diCQA on Apoptotic Signaling Pathway. The above observations confirmed that 3,5-diCQA significantly ameliorates learning and memory deficits associated with TMT-induced amnesia in mice by regulating cholinergic molecules, biochemical antioxidants, and mitochondrial activity. To investigate the cytotoxic mechanism of TMT, changes in apoptotic signaling molecules were analyzed by western blotting. We found that the ratio of phosphorylatedAkt (p-Akt)/Akt in the TMT group was drastically lower than in the vehicle control group. However, the ratio in the 3,5-diCQA group was higher than that in TMT group (Figure $7(\mathrm{a})$ ). Akt activity is influenced by numerous pathways related to various types of oxidative stress. Akt is activated by phosphorylation at Thr 308 and Ser 473, and Akt activity can be blocked by the corresponding phosphatase. The degree of Akt activity is an important factor in the improvement of neurodegenerative disorders. Akt prevents apoptosis and promotes cell survival through phosphorylation of glycogen synthase kinase- $3 \beta$ (GSK-3 $\beta$ ), BAD, and caspase 9 [36]. The activation of GSK-3 $\beta$ is responsible for abnormal hyperphosphorylation of tau, the microtubulelinked protein in neurons [37]. If GSK-3 $\beta$ is activated, apoptosis occurs as a result of increase of p-tau. Accordingly, the TMT group exhibited increased levels of $\mathrm{p}$-tau compared 


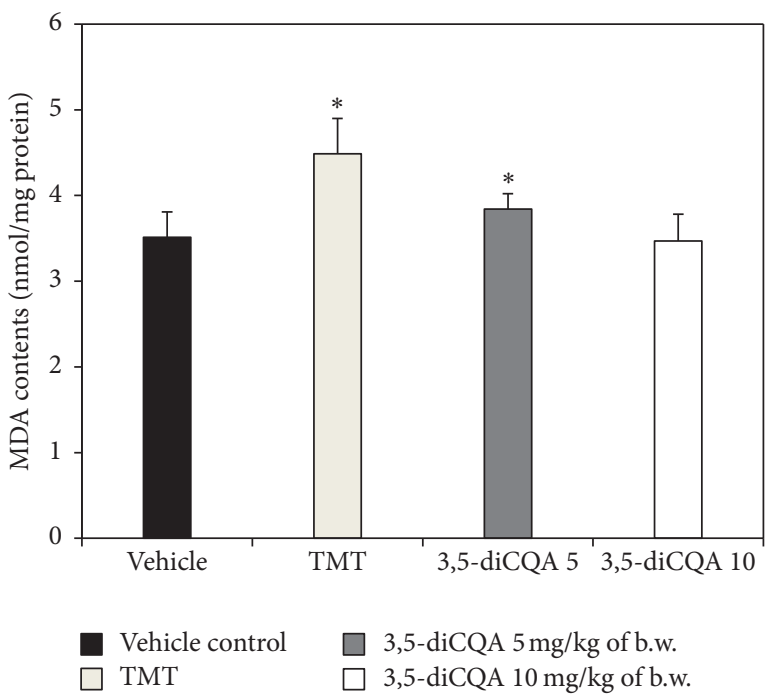

(a)

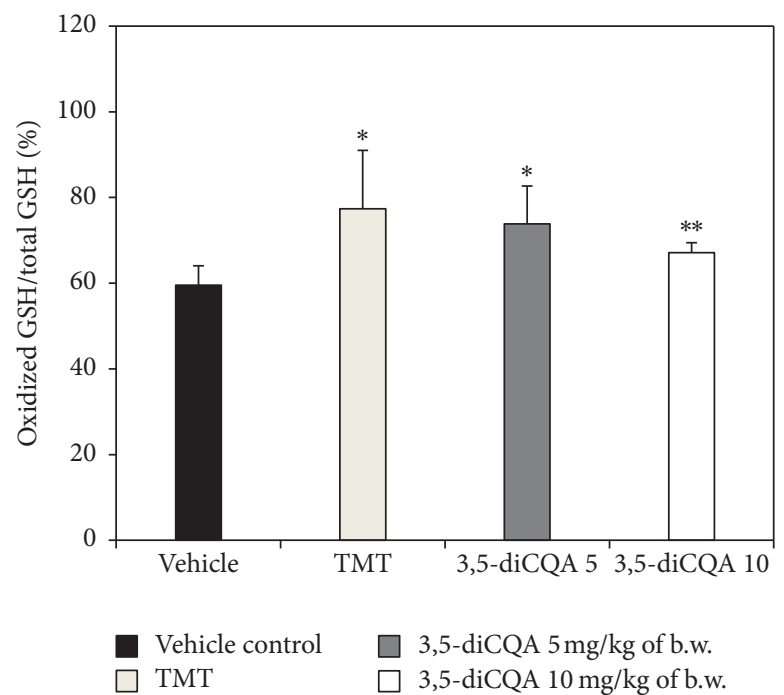

(b)

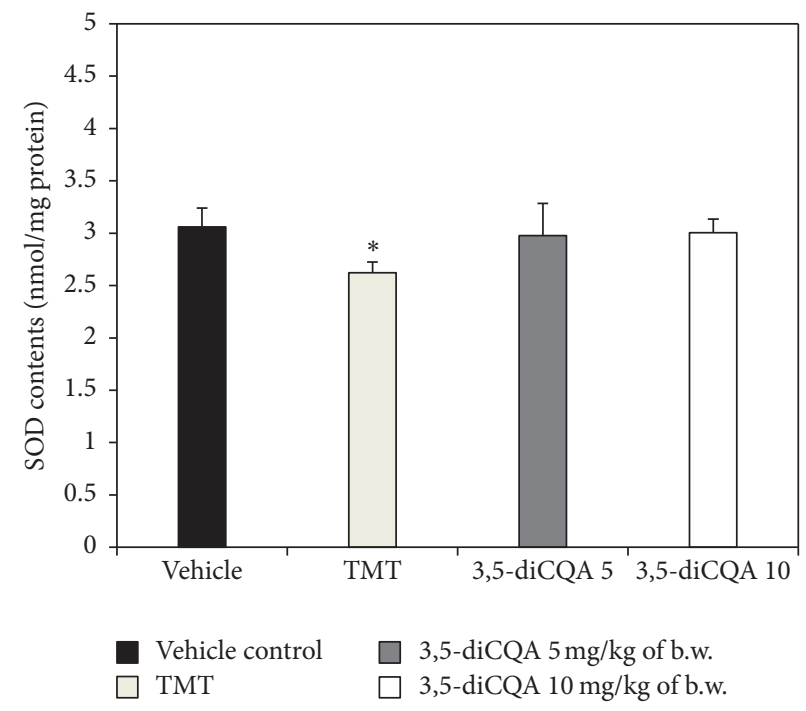

(c)

FIGURE 5: Effect of 3,5-diCQA on antioxidant biochemicals in TMT injected mice brain. MDA contents (a) and oxidized GSH/total GSH ratio (b) and total SOD contents (c). Data shown represent means $\pm \mathrm{SD}(n=8) .{ }^{*} P<0.05$ and ${ }^{* *} P<0.01$ compared to the vehicle control group.

to the vehicle control group. In contrast, the 3,5-diCQA 10 group, in which expression of p-Akt was higher than in the TMT group, showed similar expression levels as the vehicle control group (Figure 7(b)).

According to research by Zhang et al., TMT led to timeand concentration-dependent apoptotic cell death, which was associated with BAX [33]. TMT stimulated BAX expression, which leads to a loss of mitochondrial membrane potential and the release of cytochrome $\mathrm{c}$ from the mitochondria into the cytosol, which then activates the caspase protease cascade to execute apoptosis. Accordingly, the TMT group in our experiment also showed increased expression levels of BAX and a release of mitochondrial cytochrome $\mathrm{c}$ compared to the vehicle control group (Figures $7(\mathrm{c})$ and $7(\mathrm{~d})$ ). However, in the 3,5-diCQA 10 group BAX expression and the release of mitochondrial cytochrome $c$ were lower than in the TMT group. After investigating the neuroprotective effect of 1,5-diCQA against amyloid $\beta_{1-42}$-induced neurotoxicity in primary neuronal culture, Xiao et al. reported that $1,5-$ diCQA protects against neurotoxicity through activation of PI3 K/Akt followed by stimulation of Trk A and, subsequently, inhibition of GSK $3 \beta$ as well as modulation of Bcl2/BAX [38]. These results suggest that diCQAs and their isomers may be able to prevent neuronal cell death through the regulation of apoptotic signaling molecules such as Akt, BAX, and tau.

In summary, 3,5-diCQA reduced TMT-induced neuronal cell death through the activation of Akt and downregulation 


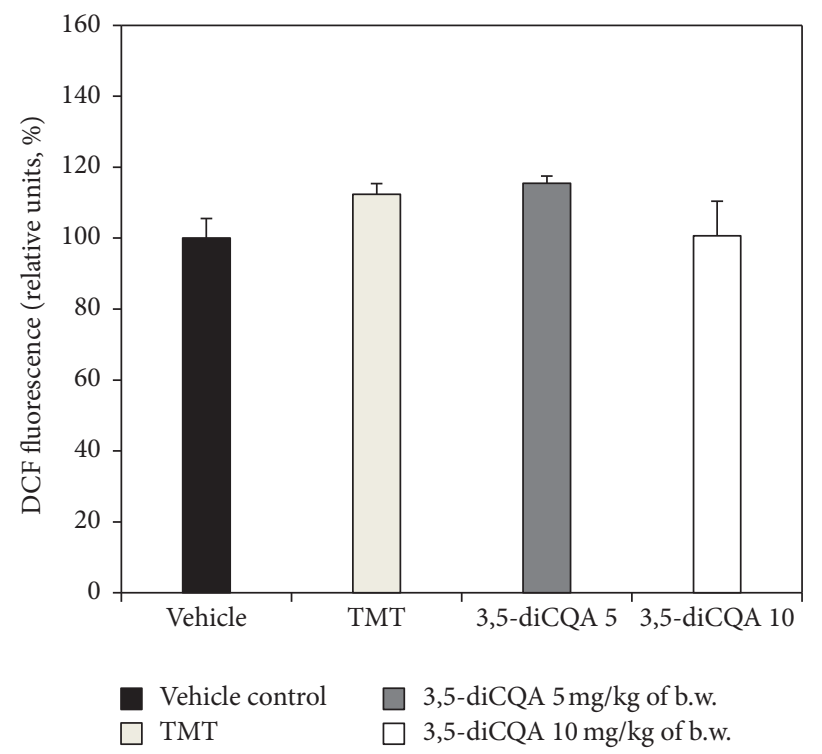

(a)

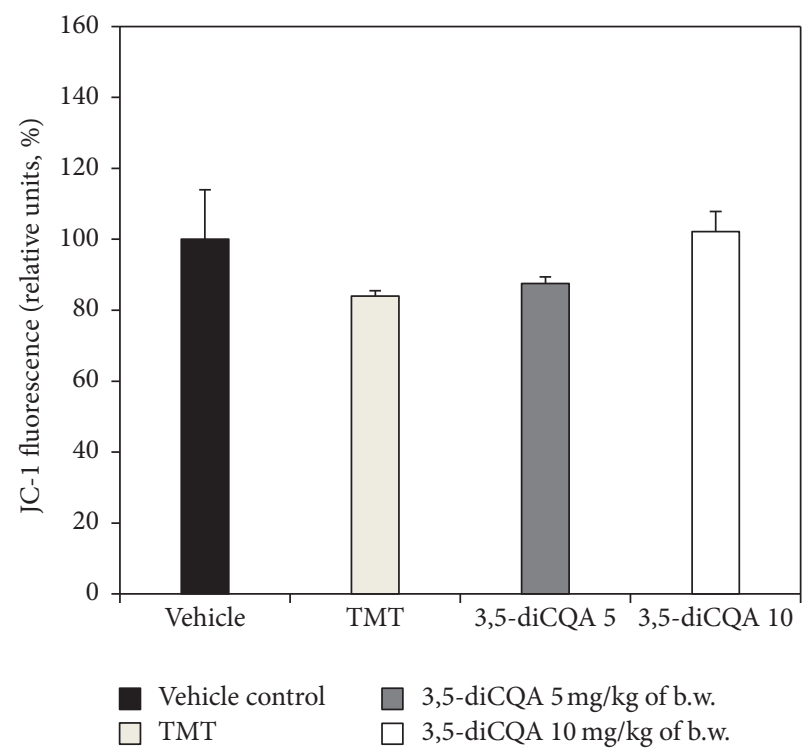

(b)

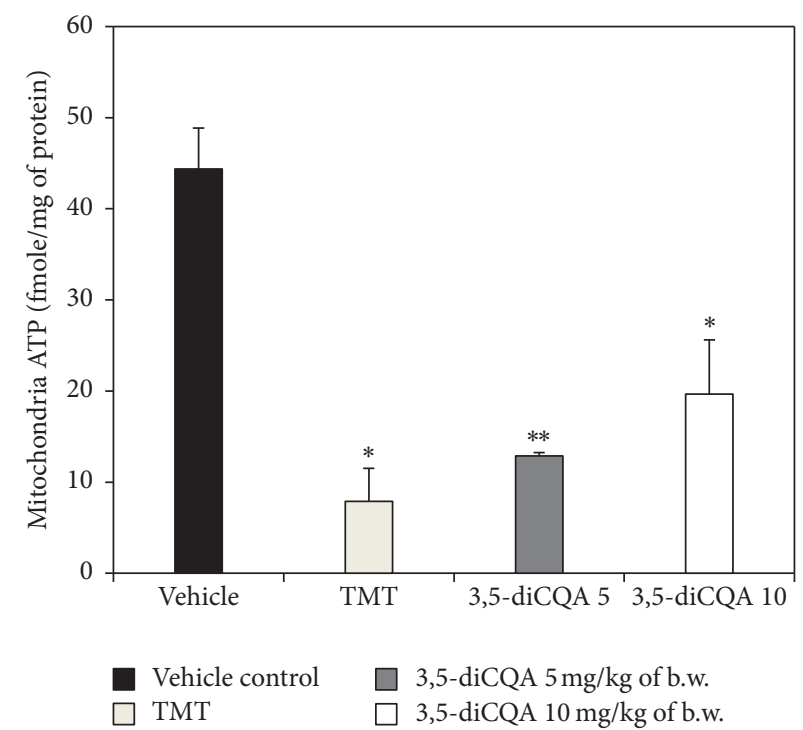

(c)

FiguRE 6: Effect of 3,5-diCQA on mitochondrial activities of TMT injected mice brain. ROS production (a), mitochondrial membrane potential (b), and ATP level (c). Data shown represent means \pm SD $(n=5) .{ }^{*} P<0.05$ and ${ }^{* *} P<0.01$ compared to the vehicle control group.

of BAX expression. Consequently, the antiamnesic effect of 3,5-diCQA, as an isomer of chlorogenic acid, with respect to cognitive dysfunction caused by TMT may be improved through neuronal cell protection through the regulation of apoptotic signaling molecules.

\section{Conclusion}

The antiamnesic effect of a 3,5-diCQA on TMT-induced learning and memory impairment in ICR mice was investigated. 3,5-diCQA, as an isomer of chlorogenic acid, significantly ameliorated cognitive dysfunction due to severe TMTinduced neurotoxicity. AChE inhibition by 3,5-diCQA went hand in hand with the recovery of ACh, a neurotransmitter in cholinergic system. In addition, the outstanding antioxidant activity of 3,5-diCQA protected against the neuronal deficit caused by excessive oxidative stress, increased oxidized GSH level, and decreased total SOD content in the brain. Mitochondrial activities, including oxidative stress, mitochondrial membrane potential, and ATP production, were also protected by 3,5-diCQA. Finally, the antiamnesic effect of 3,5-diCQA was confirmed through its regulation of apoptotic signaling molecules such as Akt, tau, BAX, and cytochrome $c$. The results suggest that 3,5-diCQA, as an isomer of chlorogenic acid, may be effective in ameliorating the cognitive impairment induced by TMT, and it seems to 


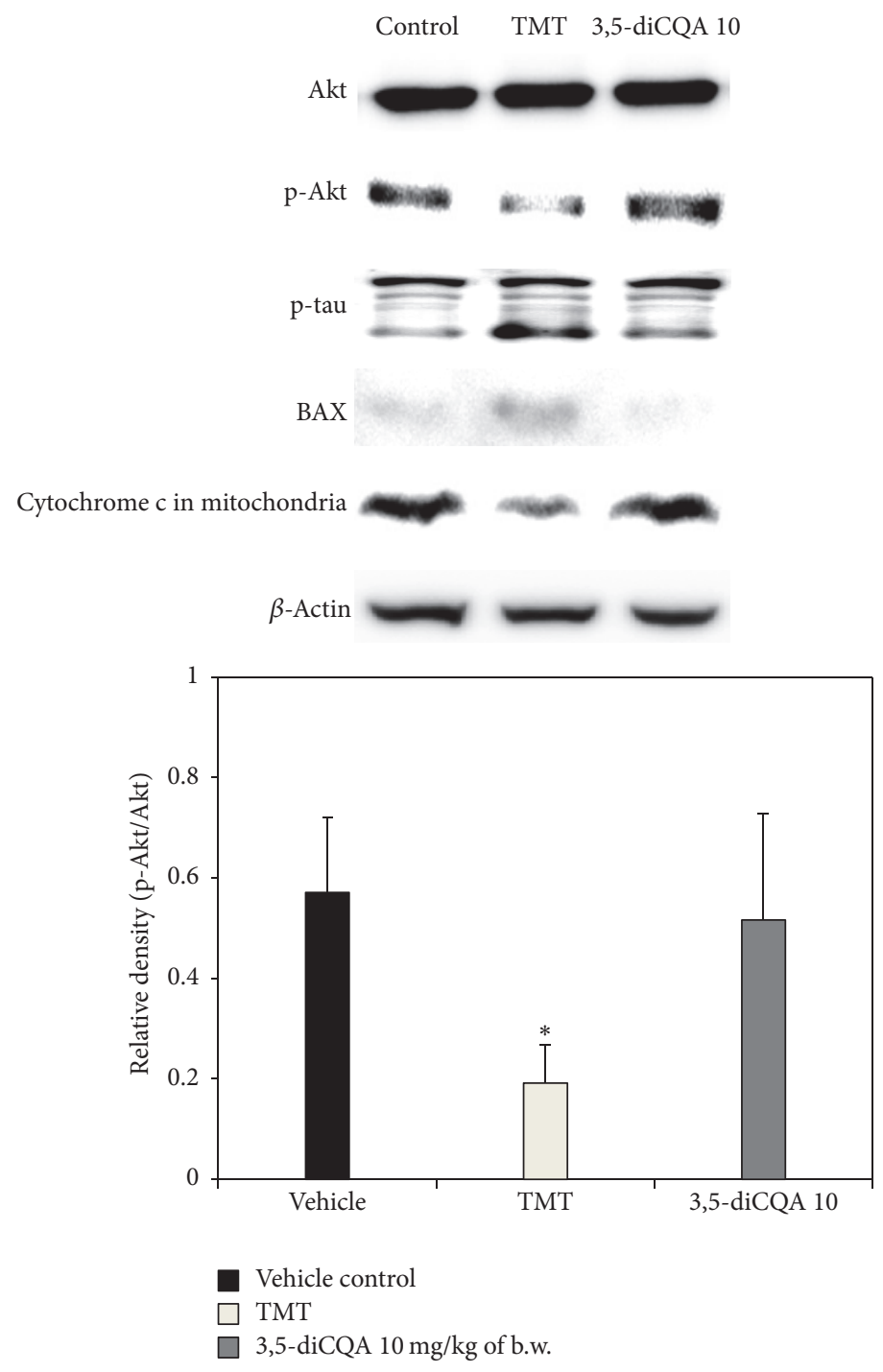

(a)
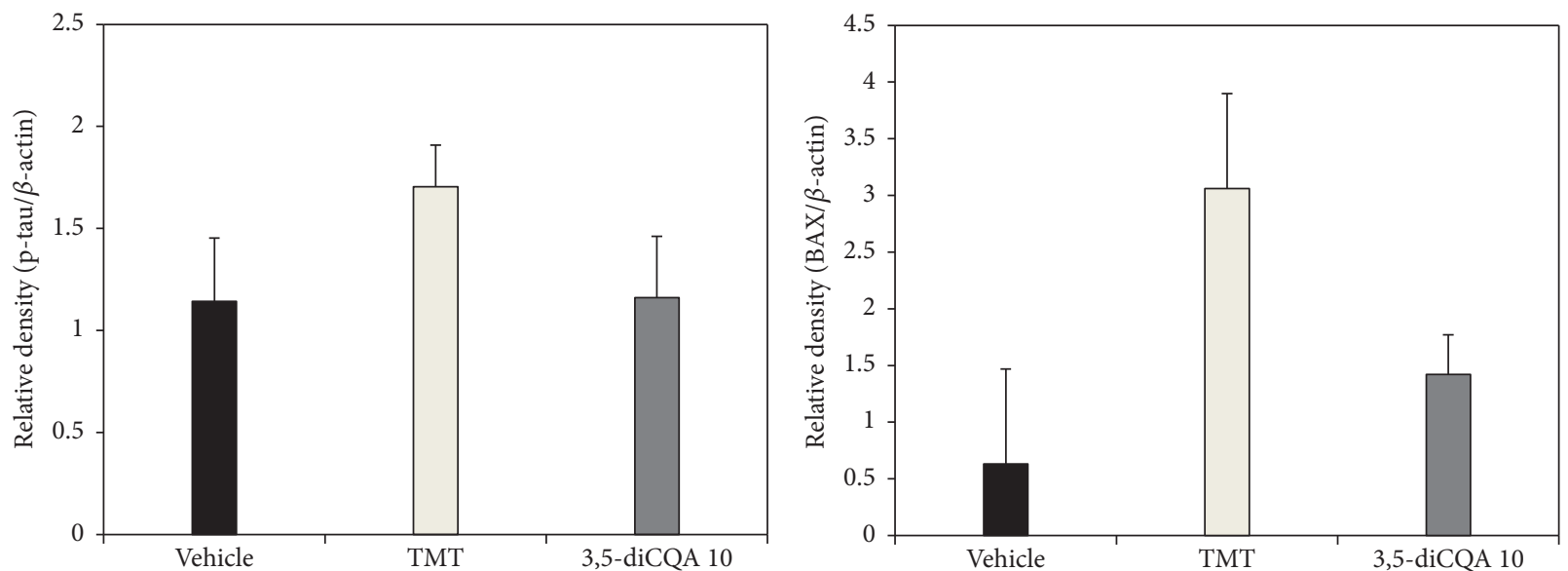

Vehicle control

$\square$ TMT

$\square$ 3,5-diCQA $10 \mathrm{mg} / \mathrm{kg}$ of b.w.

(b)

- Vehicle control

$\square$ TMT

$\square$ 3,5-diCQA $10 \mathrm{mg} / \mathrm{kg}$ of b.w.

(c)

FIGURE 7: Continued. 


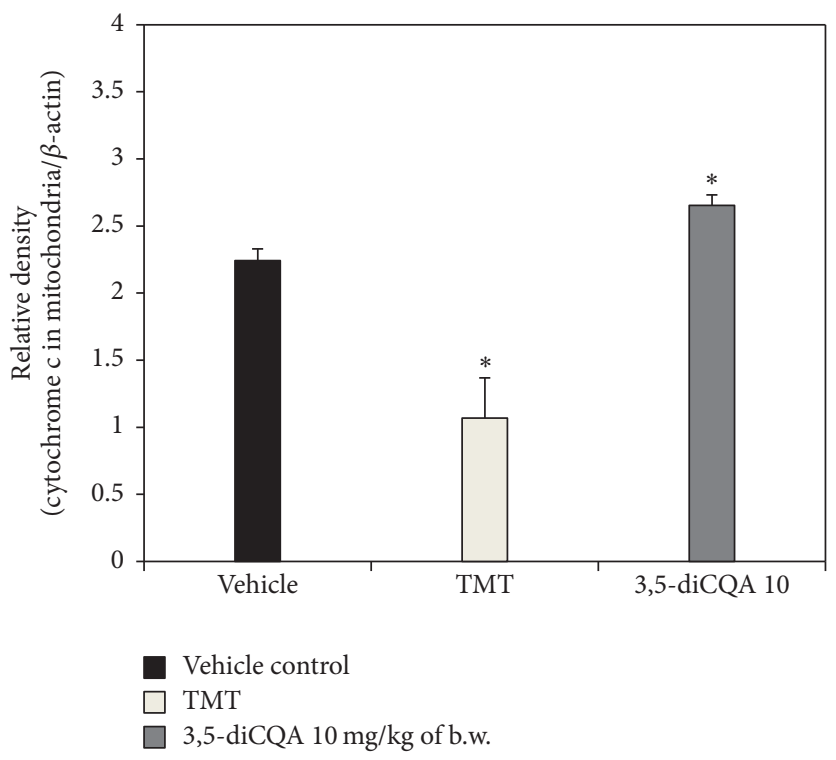

(d)

FIGURE 7: Effect of 3,5-diCQA on the expression of apoptotic signaling molecules in TMT injected mice brain. p-Akt/Akt (a), p-tau/ $\beta$-actin (b), $\mathrm{BAX} / \beta$-actin (c), and cytochrome $\mathrm{c}$ in mitochondria/ $\beta$-actin (d). Data shown represent means $\pm \operatorname{SD}(n=6) .{ }^{*} P<0.05$ compared to the vehicle control group.

be a possible candidate molecule for tackling neurodegeneration.

\section{Competing Interests}

The authors declare no competing financial interests.

\section{Acknowledgments}

This study was supported by Basic Science Research Program through the National Research Foundation of Korea (NRF2015R1D1A3A01015931) funded by the Ministry of Education, Republic of Korea. Jin Yong Kang, Seon Kyeong Park, Tian Jiao Guo, Jeong Su Ha, Du Sang Lee, and Jong Min Kim were supported by the BK21 Plus program, Ministry of Education, Republic of Korea.

\section{References}

[1] Y. Zhao and B. Zhao, "Oxidative stress and the pathogenesis of Alzheimer's disease," Oxidative Medicine and Cellular Longevity, vol. 2013, Article ID 316523, 10 pages, 2013.

[2] H. Sies, "Oxidative stress: oxidants and antioxidants," Experimental Physiology, vol. 82, no. 2, pp. 291-295, 1997.

[3] B. Halliwell and J. M. C. Gutteridge, "Oxygen toxicity, oxygen radicals, transition metals and disease," Biochemical Journal, vol. 219, no. 1, pp. 1-14, 1984.

[4] D.-Y. Choi, Y.-J. Lee, J. T. Hong, and H.-J. Lee, "Antioxidant properties of natural polyphenols and their therapeutic potentials for Alzheimer's disease," Brain Research Bulletin, vol. 87, no. 2-3, pp. 144-153, 2012.

[5] R. Stocker, "Dietary and pharmacological antioxidants in atherosclerosis," Current Opinion in Lipidology, vol. 10, no. 6, pp. 589-598, 1999.
[6] J. Chen, S. Mangelinckx, L. Ma, Z. Wang, W. Li, and N. De Kimpe, "Caffeoylquinic acid derivatives isolated from the aerial parts of Gynura divaricata and their yeast $\alpha$-glucosidase and PTP1B inhibitory activity," Fitoterapia, vol. 99, pp. 1-6, 2014.

[7] G. Baeza, M. Amigo-Benavent, B. Sarriá, L. Goya, R. Mateos, and L. Bravo, "Green coffee hydroxycinnamic acids but not caffeine protect human HepG2 cells against oxidative stress," Food Research International, vol. 62, pp. 1038-1046, 2014.

[8] M. Yoshimoto, S. Yahara, S. Okuno, M. S. Islam, K. Ishiguro, and O. Yamakawa, "Antimutagenicity of mono-, di-, and tricaffeoylquinic acid derivatives isolated from sweetpotato (Ipomoea batatas L.) leaf," Bioscience, Biotechnology and Biochemistry, vol. 66, no. 11, pp. 2336-2341, 2002.

[9] J. Y. Kim, J.-Y. Cho, Y.-K. Ma et al., "Dicaffeoylquinic acid derivatives and flavonoid glucosides from glasswort (Salicornia herbacea L.) and their antioxidative activity," Food Chemistry, vol. 125, no. 1, pp. 55-62, 2011.

[10] A. M. Abeysekera, K. T. D. De Silva, S. R. P. De Silva et al., "Inhibition of chemiluminescence generated by zymosan-activated polymorphonuclear leucocytes by phenolic constituents of Vernonia cinerea," Fitoterapia, vol. 70, no. 3, pp. 317-319, 1999.

[11] S.-S. Kim, R.-Y. Park, H.-J. Jeon, Y.-S. Kwon, and W. Chun, "Neuroprotective effects of 3,5-dicaffeoylquinic acid on hydrogen peroxide-induced cell death in SH-SY5Y cells," Phytotherapy Research, vol. 19, no. 3, pp. 243-245, 2005.

[12] S. F. Ali, C. P. LeBel, and S. C. Bondy, "Reactive oxygen species formation as a biomarker of methylmercury and trimethyltin neurotoxicity," NeuroToxicology, vol. 13, no. 3, pp. 637-648, 1992.

[13] H. J. Heo, S. J. Choi, S.-G. Choi, D.-H. Shin, J. M. Lee, and C. Y. Lee, "Effects of banana, orange, and apple on oxidative stressinduced neurotoxicity in PC12 cells," Journal of Food Science, vol. 73, no. 2, pp. H28-H32, 2008.

[14] S. J. Choi, M. J. Kim, H. J. Heo et al., "Ameliorative effect of 1,2-benzenedicarboxylic acid dinonyl ester against amyloid $\beta$ 
peptide-induced neurotoxicity," Amyloid, vol. 16, no. 1, pp. 1524, 2009.

[15] R. Morris, "Developments of a water-maze procedure for studying spatial learning in the rat," Journal of Neuroscience Methods, vol. 11, no. 1, pp. 47-60, 1984.

[16] D. Vincent, G. Segonzac, and M. C. Vincent, "Colorimetric determination of acetylcholine by the Hestrin hydroxylamine reaction and its application in pharmacy," Annales pharmaceutiques françaises, vol. 16, no. 3, pp. 179-185, 1958.

[17] G. L. Ellman, K. D. Courtney, V. Andres Jr., and R. M. Featherstone, "A new and rapid colorimetric determination of acetylcholinesterase activity," Biochemical Pharmacology, vol. 7, no. 2, pp. 88-95, 1961.

[18] J. Lu, Y.-L. Zheng, D.-M. Wu, L. Luo, D.-X. Sun, and Q. Shan, "Ursolic acid ameliorates cognition deficits and attenuates oxidative damage in the brain of senescent mice induced by Dgalactose," Biochemical Pharmacology, vol. 74, no. 7, pp. 10781090, 2007.

[19] N. Dragicevic, M. Mamcarz, Y. Zhu et al., "Mitochondrial amyloid- $\beta$ levels are associated with the extent of mitochondrial dysfunction in different brain regions and the degree of cognitive impairment in Alzheimer's transgenic mice," Journal of Alzheimer's Disease, vol. 20, supplement 2, pp. S535-S550, 2010.

[20] M. R. Brown, J. W. Geddes, and P. G. Sullivan, "Brain regionspecific, age-related, alterations in mitochondrial responses to elevated calcium," Journal of Bioenergetics and Biomembranes, vol. 36, no. 4, pp. 401-406, 2004.

[21] G. H. Doherty, D. Beccano-Kelly, S. D. Yan, F. J. Gunn-Moore, and J. Harvey, "Leptin prevents hippocampal synaptic disruption and neuronal cell death induced by amyloid $\beta$," Neurobiology of Aging, vol. 34, no. 1, pp. 226-237, 2013.

[22] C. Thornton, N. J. Bright, M. Sastre, P. J. Muckett, and D. Carling, "AMP-activated protein kinase (AMPK) is a tau kinase, activated in response to amyloid $\beta$-peptide exposure," Biochemical Journal, vol. 434, no. 3, pp. 503-512, 2011.

[23] J.-Y. Zhang, Q. Zhang, N. Li, Z.-J. Wang, J.-Q. Lu, and Y.-J. Qiao, "Diagnostic fragment-ion-based and extension strategy coupled to DFIs intensity analysis for identification of chlorogenic acids isomers in Flos Lonicerae Japonicae by HPLC-ESIMSn," Talanta, vol. 104, pp. 1-9, 2013.

[24] J. Kim, M. Yang, S.-H. Kim et al., "Possible role of the glycogen synthase kinase-3 signaling pathway in trimethyltin-induced hippocampal neurodegeneration in mice," PLoS ONE, vol. 8, no. 8, Article ID e70356, 2013.

[25] N. R. Sims, D. M. Bowen, S. J. Allen et al., "Presynaptic cholinergic dysfunction in patients with dementia," Journal of Neurochemistry, vol. 40, no. 2, pp. 503-509, 1983.

[26] M. L. Woodruff and R. H. Baisden, "Exposure to trimethyltin significantly enhances acetylcholinesterase staining in the rat dentate gyrus," Neurotoxicology and Teratology, vol. 12, no. 1, pp. 33-39, 1990.

[27] S.-H. Kwon, H.-K. Lee, J.-A. Kim et al., "Neuroprotective effects of chlorogenic acid on scopolamine-induced amnesia via antiacetylcholinesterase and anti-oxidative activities in mice," European Journal of Pharmacology, vol. 649, no. 1-3, pp. 210-217, 2010.

[28] N. Tabet, "Acetylcholinesterase inhibitors for Alzheimer's disease: anti-inflammatories in acetylcholine clothing!," Age and Ageing, vol. 35, no. 4, pp. 336-338, 2006.

[29] O. Zitka, S. Skalickova, J. Gumulec et al., "Redox status expressed as GSH:GSSG ratio as a marker for oxidative stress in paediatric tumour patients," Oncology Letters, vol. 4, no. 6, pp. 1247-1253, 2012.

[30] T. M. Al-Qirim, M. Shahwan, K. R. Zaidi, Q. Uddin, and N. Banu, "Effect of khat, its constituents and restraint stress on free radical metabolism of rats," Journal of Ethnopharmacology, vol. 83, no. 3, pp. 245-250, 2002.

[31] W. R. Markesbery, R. J. Kryscio, M. A. Lovell, and J. D. Morrow, "Lipid peroxidation is an early event in the brain in amnestic mild cognitive impairment," Annals of Neurology, vol. 58, no. 5, pp. 730-735, 2005.

[32] D. B. Zorov, M. Juhaszova, and S. J. Sollott, "Mitochondrial ROS-induced ROS release: an update and review," Biochimica et Biophysica Acta-Bioenergetics, vol. 1757, no. 5-6, pp. 509-517, 2006.

[33] L. Zhang, L. Li, K. Prabhakaran, J. L. Borowitz, and G. E. Isom, "Trimethyltin-induced apoptosis is associated with upregulation of inducible nitric oxide synthase and Bax in a hippocampal cell line," Toxicology and Applied Pharmacology, vol. 216, no. 1, pp. 34-43, 2006.

[34] D. R. Green and J. C. Reed, "Mitochondria and apoptosis," Science, vol. 281, no. 5381, pp. 1309-1312, 1998.

[35] K. S. Bhullar and H. P. Vasantha Rupasinghe, "Polyphenols: multipotent therapeutic agents in neurodegenerative diseases," Oxidative Medicine and Cellular Longevity, vol. 2013, Article ID 891748, 18 pages, 2013.

[36] K. Maiese, Z. Z. Chong, S. Wang, and Y. C. Shang, "Oxidant stress and signal transduction in the nervous system with the PI 3-K, Akt, and mTOR cascade," International Journal of Molecular Sciences, vol. 13, no. 11, pp. 13830-13866, 2012.

[37] K. Imahori and T. Uchida, "Physiology and pathology of tau protein kinases in relation to Alzheimer's disease," The Journal of Biochemistry, vol. 121, no. 2, pp. 179-188, 1997.

[38] H.-B. Xiao, X. Cao, L. Wang et al., "1,5-dicaffeoylquinic acid protects primary neurons from amyloid $\beta$ 1-42-induced apoptosis via PI3K/Akt signaling pathway," Chinese Medical Journal, vol. 124, no. 17, pp. 2628-2635, 2011. 


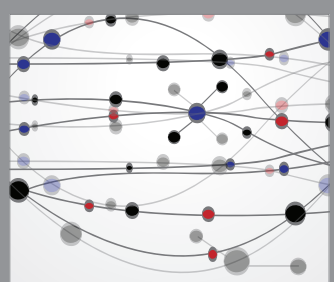

The Scientific World Journal
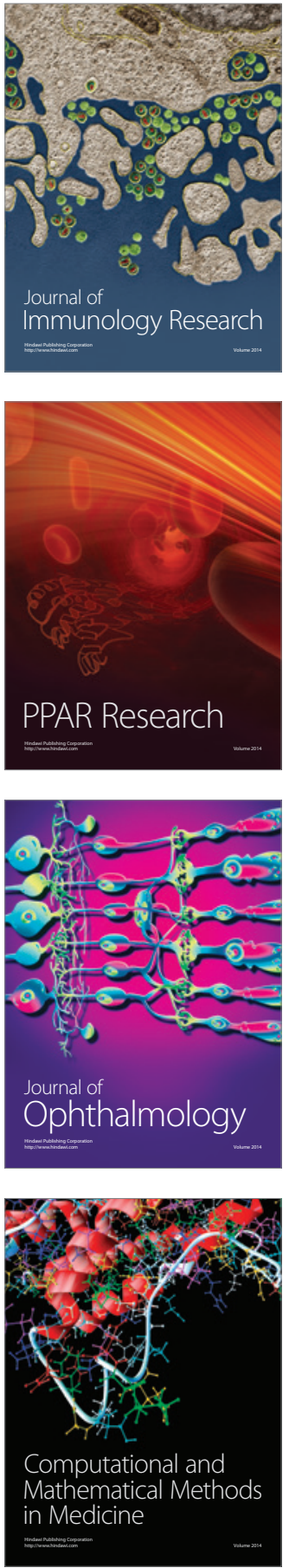

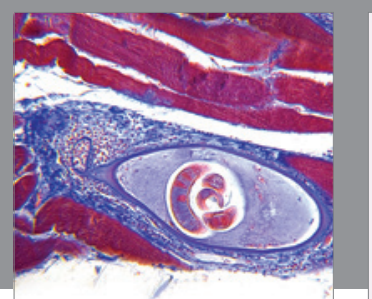

Gastroenterology Research and Practice

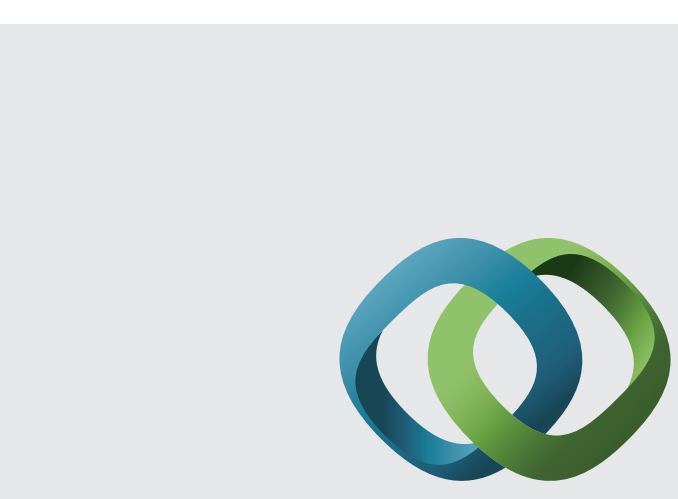

\section{Hindawi}

Submit your manuscripts at

http://www.hindawi.com
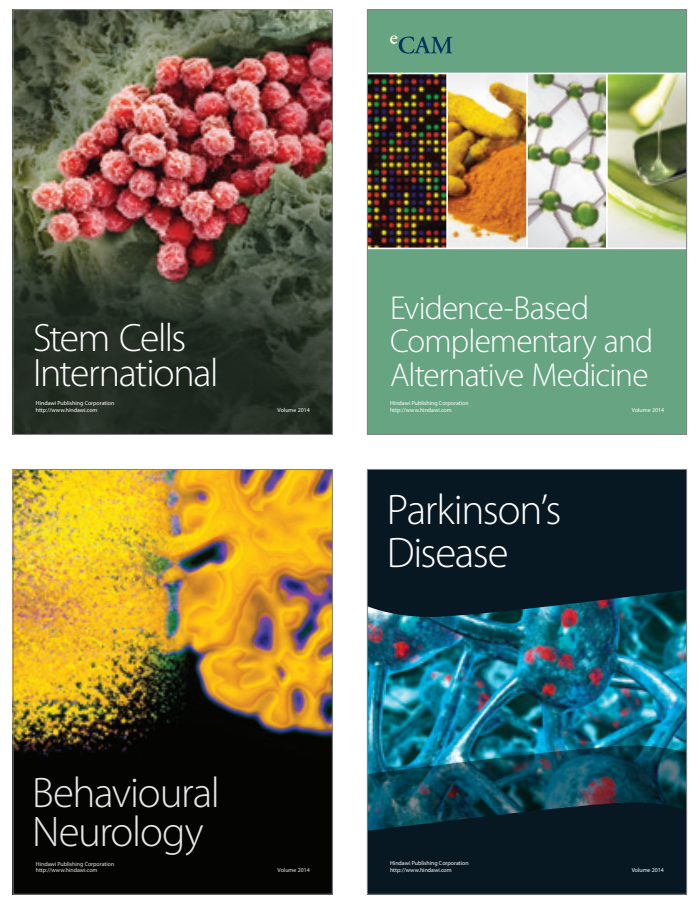
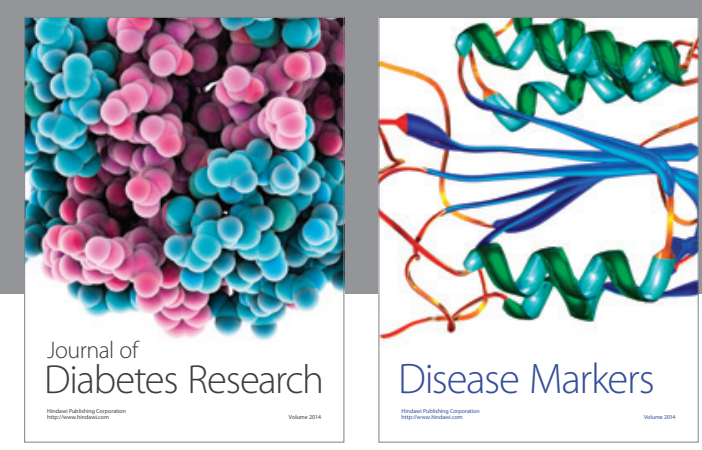

Disease Markers
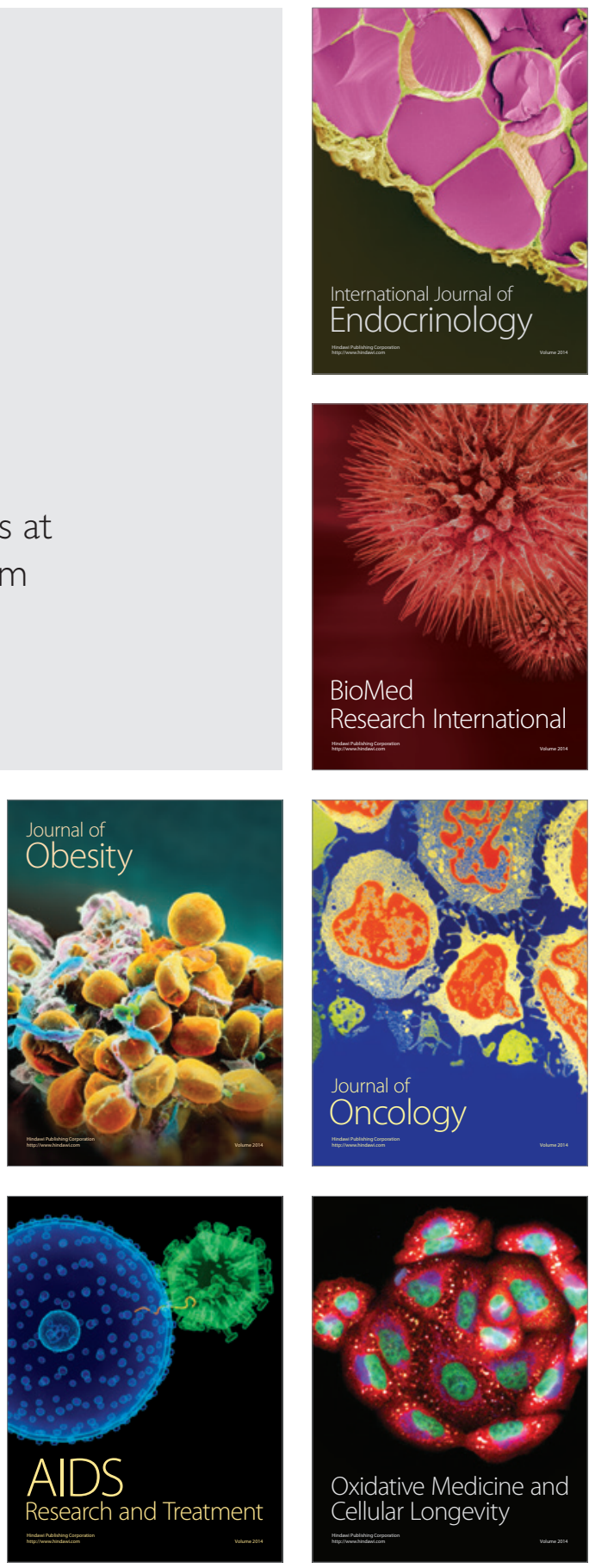